\title{
Contribution des atteintes gastro-intestinales dans le développement du syndrome de défaillance multi-viscérale radio-induit
}

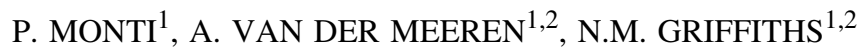

(Manuscrit reçu le 20 juillet 2005, accepté le 28 octobre 2005)

RÉSUMÉ Une atteinte sévère au niveau de la sphère gastro-intestinale, consécutive à une exposition aux rayonnements ionisants à forte dose, est un risque majeur et peut impliquer le pronostic vital de la personne irradiée. En effet, de par sa radiosensibilité importante, l'intestin représente une cible directe lors d'une irradiation, mais peut également être affecté de façon indirecte via les lésions qui apparaissent au niveau d'autres organes, comme les brûlures cutanées ou l'atteinte du système hématopoïétique. De façon similaire à ce qui peut être observé dans d'autres cas d'agressions sévères du tractus gastro-intestinal, l'exposition aux rayonnements ionisants se caractérise notamment par la perte d'intégrité de la barrière intestinale qui peut entraîner, voire perpétuer la libération de médiateurs inflammatoires et/ou anti-inflammatoires ; ce type de réponse pourrait à son tour générer des atteintes au niveau d'autres organes. Dans ce contexte, l'intestin « lésé » peut être considéré comme jouant un rôle clé dans la mise en place du syndrome de défaillance multi-viscérale (SDMV) radio-induit. La spécificité des effets de l'irradiation repose sur le fait que les compartiments tissulaires et vasculaires sont conjointement altérés avec une aplasie cellulaire plus ou moins intense, une atteinte de la fonction de barrière et la présence d'un état inflammatoire ; ainsi, tous ces éléments doivent être pris en considération dans la réponse radio-induite de l'intestin, afin de mieux déterminer le rôle de cette dernière dans la pathologie mixte du syndrome aigu d'irradiation (SAI) à court et à long terme.

ABSTRACT The contribution of radiation-induced injury to the gastrointestinal tract in the development of multi-organ dysfunction syndrome or failure.

Severe damage to the gastrointestinal tract (GIT), such as loss of mucosa and haemorrhaging following accidental overexposure to ionising radiation, appear to be a determinant feature in patient mortality. Injury to the GIT may be direct since this tissue is particularly radiation sensitive and indirect as a result of radiation burns and bone marrow aplasia. Similar to other severe trauma situations such as thermal burns or hemorrhagic shock, radiation exposure results in reduced intestinal barrier integrity which initiates and/or perpetuates inflammatory-anti-inflammatory mediator release. This may result in damage to distant organs. In this context the injured gut may be considered as a "key player" or "motor" in the development of Multiple Organ Dysfunction Syndrome or Failure (MODS/MOF). Of note is that

1 IRSN/DRPH/SRBE, Laboratoire de thérapie cellulaire et radioprotection accidentelle, BP 17, 92262 Fontenay-aux-Roses Cedex, France.

2 Adresse actuelle : CEA, DSV/DRR, Laboratoire de radiotoxicologie, BP 12, 91680 Bruyères-le-Chatel, France. 
radiation exposure elicits similar GIT injury of both mucosal and vascular elements as compared with other severe physical insults. Common factors are the intestinal inflammatory response and loss of barrier function. This radiation-induced response must be taken into account in intestinal responses after irradiation exposure in order to have a better knowledge of its implication in the mixed-pathology of the acute radiation syndrome.

Key words: radiation exposure / gastrointestinal tract / multi-organ dysfunction syndrome/failure

\section{Introduction}

Il est maintenant admis qu'une irradiation à des doses moyennes ou fortes entraîne une pathologie mixte impliquant les systèmes hématopoïétique, gastro-intestinal, neurovasculaire et cutané qui possèdent chacun un rôle majeur dans le syndrome aigu d'irradiation (SAI). L'expression de ce SAI débute quelques heures après l'exposition et peut se poursuivre jusqu'à plusieurs semaines après l'irradiation en fonction de la dose reçue (Anno et al., 1989). Le tractus gastro-intestinal fait partie des organes dits radiosensibles, le symptôme initial le plus caractéristique étant une diarrhée rebelle liée à la perte d'intégrité de la barrière intestinale (Spitzer, 1995). Cet état représente un risque majeur non seulement pour l'intestin, mais également pour l'organisme entier, et contribue fortement au pronostic vital des personnes victimes d'irradiation (Vigneulle, 1995 ; Griffiths et Lebaron-Jacobs, 2001). Selon le cas, ces atteintes gastro-intestinales peuvent résulter à la fois de l'effet direct des rayonnements ionisants, lié à la radiosensibilité de l'épithélium digestif, mais également de l'effet indirect représenté par la réponse radio-induite d'autres organes «sensibles», comme la moelle osseuse ou la peau. La communication entre différents organes, déjà reconnue sur le plan physiologique, a été de plus en plus caractérisée dans le cas de situations physiopathologiques, et plus précisément suite à différents types d'agressions sévères : c'est ainsi que des dysfonctionnements gastro-intestinaux et pulmonaires ont été mis en cause dans les conséquences de brûlures thermiques ou de choc hémorragique (Messick et al., 1994 ; Chen et al., 1999 ; Kuebler et al., 2003), dans des situations cliniques consécutives à divers traumatismes (mécaniques, chimiques, infections, ...), ou encore dans des pathologies pancréatiques ou suite à un état de choc (Deitch, 1992). La mise en place d'un continuum d'effets peut aboutir à la défaillance progressive de différents organes qui se traduit à terme par un syndrome de défaillance multi-viscérale (SDMV), traduction des termes anglo-saxons multiple organ dysfunction syndrome/failure (MODS/MOF). Le concept du «MODS » a été largement discuté dans la revue de Bone (1996). Cet auteur suggère que l'une des causes essentielles du SDMV réside dans le développement d'une forte inflammation systémique (SIRS: systemic inflammatory response syndrome) pouvant devenir néfaste si elle est incontrôlée. Cette hyper-inflammation peut être suivie dans le temps par une réponse anti-inflammatoire compensatrice 
(CARS : compensatory anti-inflammatory response syndrome) de l'organisme visant à contrôler cette inflammation, mais pouvant provoquer une immunosuppression sévère associée à un risque important d'infection tardive. Des poussées simultanées inflammatoires et immunosuppressives peuvent se développer et définissent le syndrome de réponse antagoniste mixte (MARS : mixed antagonist response syndrome). La dernière étape du SDMV serait un état de «dissonance immunologique » avec la persistance et l'emballement d'un état inflammatoire ou immunodéprimé, ne permettant pas de retour possible vers l'homéostasie, et par conséquent qui serait incompatible avec la survie de l'individu (Fig. 1).

L'intestin semble jouer un rôle essentiel dans la mise en place du SDMV via plusieurs phénomènes: le passage d'endotoxines bactériennes d'origine intestinale dans la circulation sanguine, l'hypoperfusion sanguine précoce puis la reperfusion de l'intestin qui engendre un afflux massif de médiateurs proinflammatoires. Ces réponses spécifiques de l'intestin ont été observées suite à une lésion cutanée. De façon plus précise, plusieurs études ont ainsi montré que les brûlures thermiques cutanées peuvent induire une ischémie intestinale, une perte de la fonction de barrière épithéliale et des phénomènes d'invasion bactérienne dans le sang (Gianotti et al., 1993 ; Chen et al., 1999). En retour, il est possible que les médiateurs libérés lors de ces atteintes intestinales puissent agir sur d'autres organes via le réseau vasculaire sanguin et/ou lymphatique.

La physiopathologie de l'irradiation a longtemps été considérée comme une succession de défaillances d'un organe vital unique - moelle osseuse, intestin, système nerveux central - dont l'apparition et la gravité dépendent essentiellement de la dose reçue. Depuis peu, le concept d'une mise en place progressive de dysfonctionnements de plusieurs organes s'est affirmé dans le domaine de la radiopathologie accidentelle, avec entre autre le retour d'expérience des accidents de Nesvizh (IAEA, 1996) ou de Tokai-Mura (Hirama et al., 2003). Dans cette nouvelle approche, l'issue fatale du SAI n'est plus causée par la défaillance d'un seul organe, due à la mort cellulaire, mais est la conséquence d'atteintes de plusieurs organes interdépendants, incapables de maintenir leurs fonctions homéostasiques, et qui conduisent à terme à un syndrome de défaillances multiviscérales. Cette physiopathologie impliquant la combinaison de diverses atteintes fait que le traitement d'une victime d'irradiation accidentelle à forte dose reste très délicat. En effet, si les approches thérapeutiques du syndrome hématopoiétique se montrent de plus en plus efficaces, celles visant d'autres organes cibles tels que le système digestif, la peau ou les poumons restent toujours peu convaincants, et justifient ainsi les recherches sur de nouvelles voies thérapeutiques qui prennent en compte les lésions multi-viscérales et les réponses inflammatoires associées. Cependant, concernant la cascade d'évènements qui se met en place après 
P. MONTI et al.

Agression

- Choc hémorragique

- Brûlures

- Chirurgie abdominale

- Irradiation ???
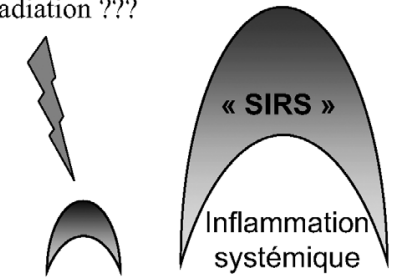

Syndrome de

Réponse

Inflammatoire

Systémique

Homéostasie systémique
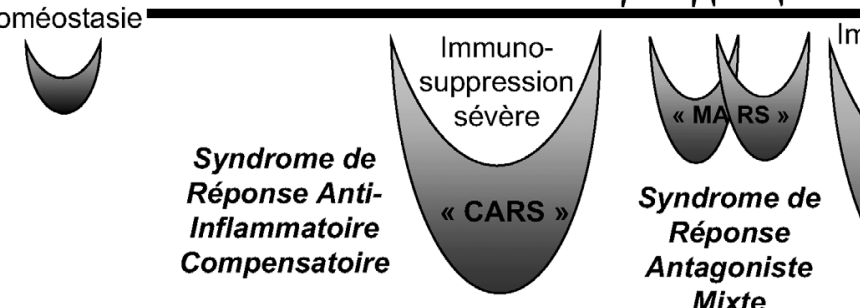

Figure 1 - Le syndrome de défaillance multi-viscérale : un concept en pleine évolution. Les différentes étapes de la mise en place du SDMV sont représentées schématiquement. À la suite d'une agression, la réponse de l'organisme s'exprime par une réaction inflammatoire locale, système de défense qui est normalement contrebalancé par une réponse anti-inflammatoire et permet de restaurer l'homéostasie. Cet équilibre est précaire et si la libération systémique d'agents proinflammatoires demeure trop importante et/ou si la réaction anti-inflammatoire ne permet pas de contrôler le phénomène, un syndrome de réponse inflammatoire systémique (SIRS) prédomine. À l'inverse, un taux excessif de médiateurs anti-inflammatoires aboutit au développement d'un syndrome de réponse anti-inflammatoire compensatoire (CARS) associé à une immunosuppression sévère. Dans certains cas, l'alternance d'une expression modérée des syndromes peut s'exprimer par un syndrome de réponse antagoniste mixte (MARS), mais si le déséquilibre persiste entre les réponses inflammatoires/anti-inflammatoires, un état de "dissonance immunologique » peut se mettre en place et correspond à l'étape ultime du SDMV (adapté de More, 1999 et Bone, 1996).

Development of multiple organ dysfunction/failure syndrome: evolving concepts. The several stages of development of MODS/MOF are presented schematically. Injury results in local release of pro-inflammatory mediators to heal wound. These are down-regulated by antiinflammatory mediators and a return to homeostasis. More severe injury involves massive local and systemic release of pro-inflammatory mediators leading to the Systemic Inflammatory response Syndrome (SIRS). A consequence may be the development of a Compensatory Antiinflammatory Response Syndrome (CARS) which results in severe immunodepression. Oscillation between SIRS-CARS but with possible return to homeostasis/recovery is termed Mixed Antagonist Response syndrome (MARS). Finally "Immunological Dissonance" refers to the stage where the balance between pro- and anti-inflammatory mediators has been lost and corresponds to the final stages in the development of MODS/MOF where either SIRS or CARS predominate (adapted from More, 1999 and Bone, 1996). 
irradiation, de nombreuses questions restent encore posées : la pathologie mixte induite par l'irradiation peut-elle être assimilée aux autres cas de SDMV ? Les mécanismes impliqués dans le développement de ces pathologies sont-ils communs ? Quel est le rôle de l'intestin dans le SDMV radio-induit ?

L'objectif de cette revue propose de comparer la réponse radio-induite de l'intestin à celle consécutive à d'autres types d'agressions, de manière à préciser de quelle façon le système digestif peut être un élément déterminant dans le développement du SDMV radio-induit.

\section{Rôle potentiel de l'atteinte gastro-intestinale dans le développement du SDMV}

Depuis l'identification du SDMV comme un état cliniquement défini, les études portant sur un concept unificateur de cette pathologie ont été à l'origine de nombreuses discussions et de recherches (Baue, 1996). Il est vraisemblable que l'intérêt croissant pour cette thématique aille de pair avec les faibles retombées provenant de la clinique, alors que l'atteinte d'organes multiples représente de plus en plus un défi permanent pour les unités de soins intensifs. En effet, elle est la cause la plus fréquente de décès des malades admis dans ces services. La reconnaissance de ce syndrome au cours de ces dernières années tient sans doute aux progrès réalisés en réanimation précoce qui ont permis de dévoiler des séquelles à retardement chez ceux qui n'auraient pas survécu auparavant. Cependant, que les études soient basées sur des données cliniques ou bien sur des modèles animaux, la plupart d'entre elles tendent à montrer que les atteintes du tractus digestif, qu'elles soient directes ou indirectes, jouent un rôle prépondérant dans la mise en place du SDMV. Malgré tout, le consensus n'est pas encore total et quelques données viennent contredire cette idée (Tab. I).

Au début des années 1980, l'intestin était considéré comme un véritable « réservoir à infections » pouvant produire, de façon constitutive, différents microorganismes et/ou métabolites bactériens plus ou moins toxiques pour l'organisme. Le passage de bactéries dans la circulation sanguine et l'infection systémique qui en résulte ont été cités comme hypothèse principale dans le développement du SDMV, et cela a pu être démontré en cas de choc hémorragique par exemple (Kale et al., 1998 ; Tani et al., 2000). Par la suite, le système digestif a été défini comme un «organe pro-inflammatoire » produisant diverses molécules comme des cytokines, des amines biogéniques, des protéases ou des radicaux libres, dont la plupart ont des effets directs sur les fonctions gastro-intestinales mais également une action indirecte sur d'autres organes à distance via le réseau systémique ou lymphatique (Deitch et al., 1994). La flore bactérienne semblerait jouer un rôle 
TABLEAU I

Rôle du système gastro-intestinal (SGI) dans le développement du syndrome de défaillance multi-viscérale (SDMV) : le pour et le contre.

Role of the gastrointestinal tract in the development of multiple organ failure: arguments For and Against.

\begin{tabular}{|c|c|}
\hline Pour & Références \\
\hline $\begin{array}{l}\text { Perte de l'intégrité de la barrière intestinale favorable au passage } \\
\text { des bactéries dans le sang }\end{array}$ & Kale et al., 1998 ; Tani et al., 2000 \\
\hline Intestin $=$ organe pro-inflammatoire & Deitch et al., 1994 ; Moore, 1999 \\
\hline $\begin{array}{l}\text { Colonisation intestinale par des bactéries pouvant avoir } \\
\text { des répercussions sur d'autres organes }\end{array}$ & $\begin{array}{c}\text { Goris et al., } 1985 \text {; Ziegler } \text { et al., } 1988 \text {; } \\
\text { Moore, } 1999\end{array}$ \\
\hline $\begin{array}{l}\text { Augmentation de la perméabilité intestinale chez des patients à risque } \\
\text { (soins intensifs) }\end{array}$ & Faries et al., 1998 ; Pape et al., 1994 \\
\hline Lien établi entre pH mucosal (acidose) et pronostic vital des patients & Bion, 1999 \\
\hline $\begin{array}{l}\text { Avantage de l'apport nutritionnel par voie entérale pour améliorer } \\
\text { les résultats cliniques }\end{array}$ & Kompan et al., 1999 \\
\hline \multicolumn{2}{|l|}{ Contre } \\
\hline $\begin{array}{l}\text { Pas de présence de bactéries ou d'endotoxines dans le sang de la veine } \\
\text { porte }\end{array}$ & Moore et al., 1991 \\
\hline $\begin{array}{l}\text { Une décontamination sélective de l'intestin réduit les infections, } \\
\text { mais n'améliore pas la survie }\end{array}$ & Reidy et Ramsey, 1990 \\
\hline
\end{tabular}

majeur, illustré par le fait qu'une modification de la microflore entrainant une croissance bactérienne accrue, est associée à une libération massive de cytokines plasmatiques après un choc hémorragique (Guo et al., 1995). À l'inverse, certains résultats viennent affaiblir l'hypothèse de l'endotoxémie et/ou de la septicémie comme seule explication dans le développement du SDMV. Ainsi, certaines études cliniques n'ont pas pu mettre en évidence la présence d'endotoxines ou de bactéries dans le sang de la veine porte de patients reçus en unité de soins intensifs (Moore et al., 1991). Par ailleurs, une décontamination sélective au niveau intestinal ne permet pas dans tous les cas d'améliorer la survie des patients (Reidy et Ramsey, 1990). Cependant, ces résultats sont encore largement controversés et demandent à être complétés. Ainsi, l'absence de bactéries ou d'endotoxines dans le sang, comme cela a été rapporté dans les travaux de Moore et al. (1991), pourrait provenir du fait que ces composés peuvent être véhiculés par d'autres voies telles que le système lymphatique.

Mis à part les phénomènes d'invasion d'endotoxines bactériennes dans la circulation sanguine, l'hypoperfusion intestinale représente un processus physiopathologique critique qui pourrait également participer à la réponse de cet organe à diverses agressions. Il a ainsi été montré qu'une agression de type ischémie-reperfusion intestinale, pouvait générer une atteinte pulmonaire aiguë (Koike et al., 1994). En conséquence, une hypoperfusion prolongée de l'intestin 
peut représenter un élément déclencheur dans la mise en place de la réponse inflammatoire systémique et du SDMV qui apparaissent après un traumatisme (Moore, 1999). La libération de médiateurs pro-inflammatoires associée à une perméabilité intestinale augmentée, ainsi qu'une diminution de la motricité iléale et de la surface de tissu lymphoïde associé à l'intestin ont été observées dans différents modèles d'atteinte intestinale suite à une ischémie-reperfusion (Moore, 1999 ; Mallick et al., 2004).

De ce fait, plusieurs arguments convaincants renforcent le concept du rôle clé de l'intestin dans la mise en place du SDMV : (i) dans des études cliniques, il a été montré une relation entre la perte de fonction de barrière intestinale et différents états pathologiques tels que la septicémie, le syndrome de détresse respiratoire aiguë (ARDS) ou le SDMV (Goris et al., 1985 ; Ziegler et al., 1988), (ii) un apport alimentaire par voie entérale améliore le résultat clinique (Kompan et al., 1999), et enfin (iii) sur des modèles animaux de brûlures ou de choc hémorragique, on a pu mettre en évidence une association entre les composants lymphatiques récoltés après l'agression et les atteintes pulmonaires ou cardiaques subséquentes (Magnotti et al., 1999 ; Sambol et al., 2002 ; Lu et al., 2004).

\section{L'irradiation et l'atteinte multi-organes : vers un concept unificateur}

Il est important de noter que les organes les plus vraisemblablement impliqués dans le pronostic vital de l'individu sont également ceux qui sont en contact direct avec des agents d'agression externes. Depuis longtemps, l'intestin se positionne comme un des organes les plus radiosensibles par le taux de renouvellement cellulaire particulièrement rapide de son épithélium (3 à 6 jours en moyenne). De ce fait, il est reconnu que l'atteinte gastro-intestinale consécutive à une irradiation à forte dose joue un rôle important dans le pronostic vital de la personne irradiée. Différents types d'expositions accidentelles, internes ou externes, ont permis de mettre en évidence que des lésions gastro-intestinales ont contribué de façon significative à une issue fatale pour les personnes irradiées (Baranov, 1994). C'est lors de l'accident de Nesvizh (Biélorussie) en 1991, où l'atteinte intestinale a été particulièrement sévère, que fut diagnostiqué pour la $1^{\text {re }}$ fois un «syndrome d'insuffisance chronique polyorganes » (IAEA, 1996) ; cela semble être le premier cas fatal d'irradiation accidentelle où la notion d'atteinte de plusieurs organes ait été mentionnée (Tab. II).

Étant donnée la radiosensibilité importante du système hématopoḯtique et l'aplasie médullaire qui s'en suit, il est évident que les systèmes de défense 
TABLEAU II

Inventaire des accidents d'irradiation mettant en cause l'atteinte du système gastro-intestinal dans le décès des personnes irradiées.

Inventory of accidental radiation exposure with involvement of gastrointestinal injury in patient mortality.

\begin{tabular}{|c|c|c|c|}
\hline Accident & $\begin{array}{l}\text { Dose et type } \\
\text { d'irradiation }\end{array}$ & $\begin{array}{l}\text { Nombre de } \\
\text { victimes } \\
\text { (nb de décès) }\end{array}$ & Commentaires \\
\hline $\begin{array}{l}\text { Vinca } \\
1958\end{array}$ & $\begin{array}{l}\text { 6,7-13,7 Gy } \\
(\text { exposition } \gamma-\mathrm{n})\end{array}$ & $6(1)$ & $\begin{array}{c}\text { Lésions importantes à l'intestin grêle et au côlon. } \\
\text { Décès à } 32 \text { jours. }\end{array}$ \\
\hline $\begin{array}{c}\text { Chernobyl }^{a} \\
1986\end{array}$ & $\begin{array}{c}8,1-16 \mathrm{~Gy} \\
\text { (exposition } \gamma-\beta \text { ) }\end{array}$ & $11(11)$ & $\begin{array}{c}\text { Diarrhées modérées à sévères. Décès entre } 14 \text { et } 32 \text { jours } \\
\text { par brûlures, } \mathrm{SGI}^{b} \text {, « ARDS }{ }^{c} .\end{array}$ \\
\hline $\begin{array}{c}\text { Soreq } \\
1990\end{array}$ & $\begin{array}{c}\approx 11 \mathrm{~Gy} \\
(\operatorname{exposition} \gamma)\end{array}$ & $1(1)$ & $\begin{array}{l}\text { Lésions importantes au niveau de l'iléon et du côlon. } \\
\text { Diarrhées intenses du } 20^{\mathrm{e}} \text { jour jusqu'au décès. Décès à } \\
36 \text { jours par « aGVHD » }{ }^{d} \text { et infection virale }{ }^{e} \text {. }\end{array}$ \\
\hline $\begin{array}{c}\text { Nesvizh } \\
1991\end{array}$ & $\begin{array}{c}\text { 9-16 Gy } \\
\text { (exposition } \gamma \text { ) }\end{array}$ & $1(1)$ & $\begin{array}{l}\text { Lésions sévères à l'intestin. Décès à } 113 \text { jours par } \\
\text { « Polyorgan chronic insufficiency syndrome ». }\end{array}$ \\
\hline $\begin{array}{c}\text { Indiana }^{f} \\
1992\end{array}$ & $\begin{array}{l}\text { Débit : } 83,8 \mathrm{~Gy} / \mathrm{h} \text { à } \\
1,5 \mathrm{~cm} \text { de la } \\
\text { muqueuse rectale } \\
(\text { exposition } \gamma)\end{array}$ & $1(1)$ & $\begin{array}{l}\text { Rectum le plus atteint, lésions sévères à l'intestin grêle } \\
\text { et au côlon. Diarrhées continues au } 5^{\mathrm{e}} \text { jour. } \\
\text { Décès au } 6^{\mathrm{e}} \text { jour. }\end{array}$ \\
\hline $\begin{array}{c}\text { Tokai-mura }^{g} \\
1999\end{array}$ & $\begin{array}{l}\text { cas } 1 / 8,5: 5,4 \mathrm{~Gy} \\
\text { cas } 2 / 4,5: 2,9 \mathrm{~Gy} \\
(\operatorname{exposition} \gamma: \mathrm{n})\end{array}$ & $3(2)$ & $\begin{array}{l}\text { Lésions modérées à sévères au niveau de l'intestin - } \\
\text { perte de muqueuse - hémorragies digestives. Décès } \\
\text { à } 82 \text { (cas 1) et } 210 \text { (cas 2) jours par syndrome de } \\
\text { défaillance multi-viscérale. }\end{array}$ \\
\hline
\end{tabular}
contre l'hôte ; ${ }^{e}$ Cytomegalovirus ; ${ }^{f}$ Flynn et al., $1995 ;{ }^{g}$ Hirama et al., 2003.

immunitaire des tissus sont amoindris après exposition. Ce degré d'aplasie n'est pas rencontré à la suite d'autres agressions sévères de l'organisme, tels que les traumatismes mécaniques, thermiques ou d'autres pathologies organiques comme la pancréatite. Dans ces différents cas de figure, l'activité hématopoïetique est encore opérationnelle et permet la présence de cellules immunocompétentes actives au niveau systémique et tissulaire. Après irradiation, les conditions sont toutes autres puisque, très rapidement, une aplasie médullaire plus ou moins prononcée peut apparaître et perdure d'une façon variable selon la dose et la surface d'exposition. La reprise hématopoïétique post-irradiation, observée chez les accidentés de Nesvizh ou de Tokai-Mura, n'a toutefois pas permis d'éviter le décès de ces patients par SDMV. Cependant, ces résultats n'excluent pas le fait que l'aplasie initiale, puis la reprise hématopoïétique observées après irradiation restent des évènements importants à considérer pour permettre de mieux comprendre l'évolution clinique impliquant plusieurs organes. 


\section{Similitudes entre les atteintes gastro-intestinales radio-induites et celles résultant d'autres agressions}

\subsection{Atteintes de la barrière épithéliale}

L'intestin grêle et le côlon forment une barrière entre le milieu extérieur et le milieu intérieur, barrière à la fois soumise à des substances exogènes, mais également à la microflore endogène et aux différentes toxines qu'elle peut libérer. En conditions physiologiques, le premier niveau de la barrière intestinale est d'ordre physico-chimique, représenté par le $\mathrm{pH}$ luminal et la couche de mucus qui recouvre la surface épithéliale. Le second niveau, considéré comme la barrière principale, est constitué par la monocouche de cellules épithéliales qui est en renouvellement constant. Les protéines des jonctions serrées et adhérentes qui existent entre les cellules épithéliales permettent d'assurer d'une part le maintien de l'intégrité de l'épithélium et d'autre part les fonctions qui en découlent comme par exemple le passage de petites molécules $(<400 \mathrm{Da})$ via la voie de transport paracellulaire. De nombreuses études menées chez l'homme ou l'animal ont pu montrer une augmentation de la perméabilité de l'intestin grêle ou du côlon après exposition aux rayonnements ionisants (Solheim et al., 1991 ; Nejdfors et al., 2000 ; Thiagarajah et al., 2000 ; Vigneulle et al., 2002). Ces altérations sont essentiellement exprimées au cours de la phase aiguë, durant les deux premières semaines qui suivent l'irradiation et sont associées à une perte de cellules et à des modifications des jonctions intercellulaires au niveau de l'épithélium. À l'heure actuelle, on ne dispose que de rares études portant sur l'évolution de ces modifications à plus long terme. Par ailleurs, il est clairement reconnu que si l'intestin représente un organe particulièrement radiosensible, il possède également une forte capacité de récupération, structurale et fonctionnelle, tout comme le système hématopoïétique (De Both et Verney, 1976 ; Lamerton et Lord, 1964). Ce fait a pu être vérifié lors de l'autopsie de l'accidenté de Nesvizh, où à l'échelle organique comme au niveau microscopique, on a pu noter une récupération partielle de l'épithélium du système digestif, trois mois après l'irradiation (IAEA, 1996).

Cette capacité de récupération est due au fait que la majorité de cellules qui composent l'épithélium intestinal ont une activité mitotique intense et se renouvellent à partir des cellules souches situées au niveau des cryptes. De façon identique au système hématopoïétique, ce type cellulaire est particulièrement radiosensible et son niveau d'atteinte conditionnera celle du tissu considéré. Ceci fut largement étudié par Potten et son équipe qui ont pu montrer que les cellules les plus radiosensibles de l'épithélium intestinal se situent en position 4-6 dans les cryptes de l'intestin grêle et dans le tiers inférieur des cryptes coliques, 
correspondant à la zone de localisation des cellules souches intestinales. Ce résultat a été obtenu par observation sur coupes histologiques de cellules en apoptose dans les heures qui suivent l'irradiation et ce phénomène est devenu une des « signatures » des effets radio-induits sur l'intestin (Potten, 1990 ; Potten et Grant, 1998). Dans le cas d'irradiations à fortes doses et par conséquent pour des atteintes importantes au niveau des cellules épithéliales, les cellules souches survivantes sont incapables de fournir suffisamment des cellules pour repeupler les villosités, et ce phénomène aboutit à une érosion de l'épithélium intestinal avec diminution de la hauteur des villosités et altérations fonctionnelles. Ces phénomènes peuvent conduire à une perte quasi-totale de la fonction de barrière accompagnée d'une baisse des processus d'absorption de l'eau, des électrolytes et des nutriments (Potten, 1995 ; Beccioloni et al., 1995). Ainsi, l'invasion bactérienne systémique et la perte de fluides et d'électrolytes sont favorisées, et ces événements physiopathologiques décrits depuis longtemps dans le syndrome gastro-intestinal radio-induit peuvent conduire à la mort de l'individu. Par ailleurs, on sait que ces cellules souches sont également sensibles à d'autres agressions externes, comme la chimiothérapie ou d'autres toxiques chimiques.

De façon similaire aux atteintes radio-induites de l'intestin, les brûlures thermiques peuvent altérer la fonctionnalité de cet organe en réduisant l'absorption et en augmentant la perméabilité de l'épithélium. Par ailleurs, une relation a pu être établie entre les altérations de la perméabilité intestinale et le degré des brûlures (Ryan et al., 1992). Varedi et al. (1999) ont également démontré que l'ajout de sérum issu de rats brûlés sur des cultures de cellules d'origine intestinale se traduisait par une perte de l'intégrité de la monocouche cellulaire, suggérant l'effet à distance des cellules lésées sur des cellules saines (simulation d'un effet abscopal). Enfin, les mêmes auteurs ont mis en évidence une augmentation de l'apoptose ainsi qu'une réduction de la prolifération cellulaire, dans les cryptes intestinales de rats ayant subi une brûlure thermique cutanée (Varedi et al., 2001). Après agression, l'apoptose apparaît de façon précoce au niveau de localisations spécifiques de la crypte, et plus particulièrement des cellules souches de l'intestin grêle, tout comme cela a pu être observé après irradiation.

D'autres cas d'agressions sévères peuvent également moduler la perméabilité intestinale; ainsi, cette dernière est fortement augmentée après un choc hémorragique chez l'animal (Wang et al., 1998 ; Tawadrous et al., 2002) ou chez des patients (Roumen et al., 1993). À titre d'exemple, la comparaison des effets de diverses agressions est illustrée dans la figure 2. Une irradiation corps entier à forte dose chez la souris (18 Gy) génère une importante atteinte structurale de l'épithélium intestinal qui s'accompagne d'une forte augmentation de la perméabilité intestinale (Monti et al., 2004). De façon similaire, un choc septique (injection intra-péritonéale de LPS) ou un choc hémorragique peuvent générer de 
Irradiation
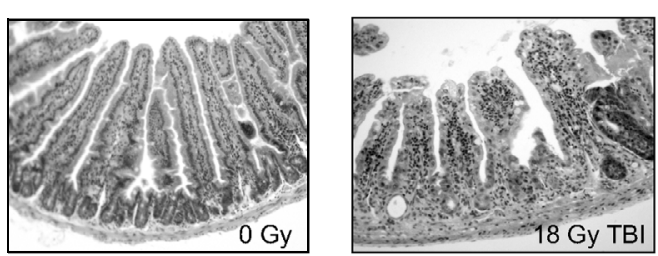

A- Morphologie jéjunale 4 jours après une irradiation corps entier de $18 \mathrm{~Gy}$ chez la souris

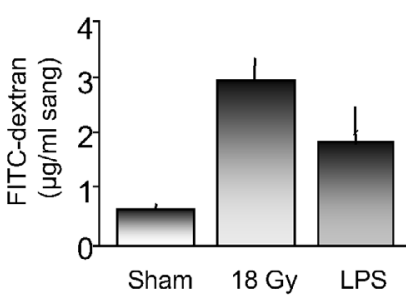

$\mathrm{B}$ - Perméabilité jéjunale après irradiation ou injection intrapéritonéale de LPS chez la souris

\section{Choc hémorragique}

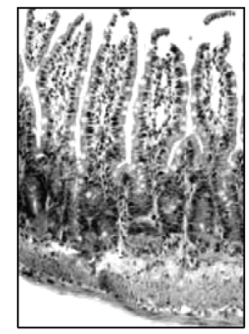

C - Morphologie intestinale après choc hémorragique chez le rat

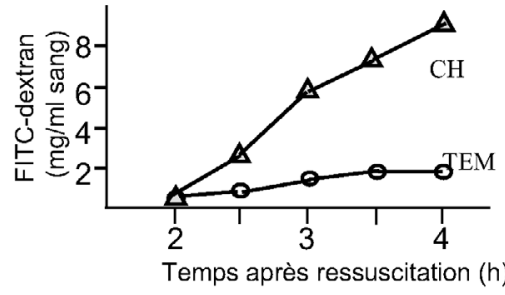

D - Permeabilité intestinale après choc hémorragique $(\mathrm{CH})$ chez le rat et chez les rats témoins (TEM)

Figure 2-Effet comparé de l'irradiation, d'un choc septique ou d'un choc hémorragique sur la morphologie et la perméabilité intestinale. Coupe histologique longitudinale de jéjunum de souris 4 jours après irradiation corps entier à la dose de 18 Gy (obj. ×10) en comparaison à celle obtenue chez des animaux témoins (A). De façon concomitante à l'atteinte structurale, la perméabilité mesurée au niveau d'une anse jéjunale (mesure du passage d'un marqueur fluorescent normalement non perméant, le FITC-dextran $4000 \mathrm{Da}$ ) est fortement augmentée, tout comme cela peut être observé après un choc septique reproduit par injection intrapéritonéale de lipopolysaccharide (LPS) (B). Après choc hémorragique, l'atteinte structurale de l'iléon de rat est également visualisable (C) (obj. x10, image prêtée par les Drs Xu et Deitch) et une augmentation de la perméabilité intestinale est observée (D) (d'après Kuebler et al., 2003). Comparison of the effects of total body irradiation, endotoxaemia or haemorrhagic shock on intestinal structure and permeability. Mouse jejunal morphology in control and irradiated (18 Gy, total body irradiation) animals (obj x10) (A). Mouse jejunal permeability of FITCdextran (4 kd) following total body irradiation (18 gy) or lipopolysaccharide (lps) injection (B). Rat ileal morphology post haemorrhagic shock ( $\times 10$ obj) (C) (after Xu and Deitch). Rat intestinal permeability of FITC-dextran $(4 \mathrm{kd})$ following haemorrhagic shock (D) (after Kuebler et al., 2003). 
telles altérations morphologiques ou fonctionnelles. Kuebler et al. (2003) ont d'autre part démontré que l'augmentation de la perméabilité intestinale se traduit par un passage facilité de molécules dans le compartiment sanguin, mais également dans la circulation lymphatique, les molécules les plus grosses (40 kDa) s'accumulant préférentiellement dans la lymphe alors qu'une répartition homogène se fait pour les molécules de plus petite taille.

La plupart des effets cellulaires observés après brûlures cutanées, choc hémorragique ou irradiation sont en partie reliés à la réponse des cellules ayant une importante activité proliférative. Étant donné la multiplicité des types cellulaires au sein de l'épithélium intestinal, il est évident que toute perte de cellules peut contribuer à diminuer la synthèse et la sécrétion de facteurs mitogènes, motogènes ou anti-microbiens qui agissent naturellement pour maintenir ou rétablir l'homéostasie de l'intestin. Â titre de conclusion, on pourrait retenir que l'apoptose des cellules épithéliales de l'intestin joue un rôle important dans la phase critique des pathologies d'origine infectieuse ou non (Husain et Coopersmith, 2003) et dans le développement de l'atteinte multi-organes (Guan et al., 2002).

\subsection{Conséquences des variations de la circulation sanguine et lymphatique}

L'irrigation de l'intestin représente un paramètre important du fait que cet organe nécessite à lui seul plus de $30 \%$ du débit cardiaque pour un fonctionnement correct. Par conséquent, la moindre altération de la vasomotricité au niveau intestinal ou sur un site plus éloigné peut avoir des répercussions dans les fonctions de l'épithélium. De ce fait, l'absorption intestinale des nutriments dépend non seulement du niveau d'activité des enzymes de l'épithélium, mais également de l'état vasculaire. En conditions physiologiques, l'intestin est soumis à des variations de l'irrigation sanguine locale par un phénomène que l'on nomme « ischémie-reperfusion », correspondant à des phases d'irrigation réduite suivies par une reprise normale du débit sanguin. Un des modèles expérimentaux classiquement utilisé pour l'étude du SDMV s'appuie sur une atteinte intestinale consécutive à une «ischémie-reperfusion » provoquée de forte intensité. Cette ischémie engendre des altérations de la barrière intestinale qui se traduisent par une augmentation de la perméabilité d'un épithélium (Bush et al., 2000).

Concernant l'exposition aux rayonnements ionisants, il ne ressort pas de façon claire si l'atteinte intestinale radio-induite résulte d'une ischémie-reperfusion prise dans le sens strict du terme, c'est-à-dire une hypoperfusion splanchnique suivie d'une reperfusion intense avec afflux de médiateurs inflammatoires, telle qu'elle est décrite par Moore (1999) dans le cadre d'autres agressions. Le consensus est encore loin d'être établi puisque certains résultats ont même montré une atténuation 
des processus physiologiques d'ischémie-reperfusion après irradiation, corrélée à une baisse du nombre de mastocytes et de neutrophiles présents dans l'épithélium intestinal (Harari et al., 2000). Cette discordance permet cependant de poser la question sur l'incidence des dommages du système hématologique et de sa récupération dans le développement du SDMV après exposition aux rayonnements ionisants.

Au niveau intestinal, les modifications structurales et fonctionnelles de la circulation sanguine mais également de l'endothélium vasculaire proprement dit ont été observées après irradiation, à partir de différentes techniques d'investigation. Ainsi, une augmentation de la perméabilité vasculaire associée à une distension des capillaires sanguins représente un des effets précoces classiquement observé après irradiation (Willoughby, 1960 ; Cockerham et al., 1984). Eddy et Casarett (1968) ont démontré par analyse histologique associée à une micro-angiographie différents types d'altérations de la microcirculation intestinale : dilatation, réduction et déformation des artères, réduction du nombre et/ou de la longueur des vaisseaux et de façon plus tardive, apparition de foyers hémorragiques. Par la suite, cette augmentation de la perméabilité vasculaire après irradiation a été confirmée à partir de différentes approches expérimentales allant de l'étude in vivo (Buell et Harding, 1989 ; Panès et al., 1995; Van der Meeren et al., 2004) à des analyses de l'ultrastructure vasculaire par microscopie électronique (Debbage et al., 2000). Des modifications de la perméabilité vasculaire vis-à-vis des molécules et/ou de cellules jouent clairement un rôle fondamental dans la mise en place de la réponse inflammatoire. Pour conforter cette hypothèse, plusieurs études expérimentales rapportent une augmentation radio-induite de la libération de divers médiateurs (cytokines pro-inflammatoires, bio-amines, monoxyde d'azote) associée à une surexpression des molécules d'adhésion au niveau intestinal (Buell et Harding, 1989 ; Panès et al., 1995 ; Freeman et Mac Naughton, 2000 ; Linard et al., 2003). Ces arguments viennent renforcer l'idée avancée précédemment mettant en avant le rôle de l'hypoperfusion intestinale dans la libération massive de médiateurs inflammatoires : ceci pourrait avoir à la fois une incidence locale mais également sur des organes distants et viendrait illustrer le rôle clé que l'intestin peut jouer dans la mise en place du SDMV.

L'origine de la lésion primaire - mort cellulaire des cellules souches de l'épithélium versus mort cellulaire des cellules endothéliales - est un sujet encore largement débattu. Depuis les travaux d'Eddy et Casarett en 1968, un nombre varié d'approches a mis en avant le rôle important de l'apoptose radio-induite des cellules endothéliales dans l'atteinte de la muqueuse intestinale et finalement dans la survie de l'individu (Haimovitz-Friedman et al., 1997 ; Paris et al., 2001 ; Maj et al., 2003; Cho et al., 2004). Il a été montré que la protection des cellules endothéliales par un traitement avec un facteur de croissance, le basic fibroblast 
growth factor (bFGF), exerce un effet positif sur la survie, mais également sur la morphologie de l'intestin de souris irradiées. Sur la base de ces résultats, les auteurs ont émis l'hypothèse que les atteintes de la microcirculation influencent celles du compartiment clonogénique des cryptes intestinales, qui à leur tour peuvent intervenir dans l'évolution des dommages radio-induits à l'intestin (Maj et al., 2003). Cependant, aucun des effets positifs du bFGF, ou de tout autre action de protection de l'endothélium, n'a été observé sur le nombre de foyers prolifératifs au sein de l'épithélium.

Le transport de médiateurs relargués par l'épithélium intestinal en réponse à une agression s'effectue par voie sanguine principalement, mais la voie lymphatique ne peut être exclue. En effet, en plus de son implication dans l'homéostasie intra- et inter-tissulaire, le réseau lymphatique de l'intestin joue un rôle actif dans la diffusion des nutriments liposolubles absorbés et, bien que son rôle précis soit encore sujet à controverse, il peut représenter une des voies importantes de communication entre les différents organes. Cette notion a pu être illustrée dans le cas d'une réponse inflammatoire, générée par brûlure thermique de la peau, dont les répercussions intestinales et pulmonaires ont pu être démontrées (Magnotti et al., 1999). Deitch et son équipe ont pour leur part largement contribué à démontrer le rôle de la lymphe en utilisant la dérivation de la circulation lymphatique ou la collecte de la lymphe dans différents modèles physiologiques : brûlure thermique, ischémie-reperfusion de l'intestin ou choc hémorragique. Ainsi, l'intervention de la voie lymphatique dans l'induction d'effets abscopaux (ou à distance), c'est-à-dire au niveau d'organes ou de cellules non soumis directement à l'agent d'agression, a été démontrée : augmentation de la perméabilité et de l'expression de molécules d'adhésion dans les poumons après brûlure thermique ou choc hémorragique (Magnotti et al., 1999, Zallen et al., 1999) ; diminution de la contractibilité au niveau cardiaque après brûlure (Sambol et al., 2002) ; augmentation des molécules d'adhésion dans des cultures de cellules endothéliales (Adams et al., 2001) et diminution de l'hématopoï̀se dans la moelle osseuse (Anjaria et al., 2001) après choc hémorragique ; augmentation de l'apoptose des cellules épithéliales et endothéliales pulmonaires après choc hémorragique (Lu et al., 2004). À partir de toutes ces données, on peut émettre l'hypothèse qu'en cas d'irradiation, un territoire hématopoïétique non exposé peut malgré tout être affecté par l'affluence de médiateurs inflammatoires provenant d'autres organes par voie lymphatique. Cette remarque est d'autant plus importante que dans la majorité des accidents d'irradiation, l'exposition est hétérogène et préserve donc a priori des zones fonctionnelles d'hématopoièse. L'exemple des accidents de Nesvizh et de Tokai-Mura montre que malgré une récupération partielle de l'hématopoïèse, le niveau des leucocytes circulants n'est jamais revenu à des valeurs normales. On peut penser que ce phénomène 
résulterait d'une libération continue d'agents, d'origine intestinale ou cutanée, et véhiculés par la lymphe, comme cela a été proposé dans le cas d'autres traumatismes sévères (Deitch, 2001, 2002). Si l'implication du drainage lymphatique est de plus en plus étudiée dans les modèles de choc ou de brûlures thermiques, le modèle de l'irradiation reste encore inexploré. Ce concept du transport lymphatique de cellules et/ou médiateurs pouvant générer des effets à distance sur d'autres organes dans des modèles de SDMV est récent (Kaiser et al., 2005) et mériterait également d'être considéré dans le domaine des expositions aux rayonnements ionisants. Nos derniers travaux ont déjà permis de mettre en évidence un effet abscopal au niveau pulmonaire après irradiation abdominale (Van der Meeren et al., 2005); la voie de communication entre l'intestin «agressé » et les poumons reste à découvrir, mais la contribution lymphatique représente un bon candidat.

\section{Conclusions}

En conditions physiologiques, le tractus digestif, la peau et le système hématopoïétique interagissent pour maintenir l'homéostasie de l'organisme. À la suite d'une irradiation à forte dose ou de la perturbation de l'un de ces systèmes, les différents mécanismes impliqués dans l'état d'équilibre sont sévèrement atteints et peuvent générer des réponses inappropriées. Il est de plus en plus évident, à partir des données des récents accidents d'irradiation ou issues d'autres modèles d'agression, que l'intestin lésé joue un rôle pivot dans la mise en place de l'atteinte multi-organes et dans le pronostic vital des patients. Les lésions directes, mais également les communications entre organes, sont à considérer dans la réponse globale radio-induite.

La question sur l'importance des cellules et/ou molécules d'origine lymphatique qui apparaissent après irradiation reste ouverte. Il est possible que même si le compartiment des cellules souches intestinales récupère en partie son activité, des facteurs secondaires reliés à l'atteinte d'autres organes pourront perpétuer et aggraver les altérations des fonctions de l'intestin et par conséquent son intégrité. Ces réponses « inadéquates » peuvent évoluer et persister avec le temps et moduler différents traitements, comme la transplantation d'éléments sanguins (transfusions sanguines, greffe de moelle osseuse) ou l'apport d'autres types de cellules (cellules souches mésenchymateuses par exemple). Ces interventions pourraient également participer à la mise en place du SDMV.

Si l'on compare les réponses du système digestif consécutives à diverses agressions sévères à celles observées après irradiation, on peut trouver des similitudes à la fois au niveau des compartiments vasculaires et tissulaires. À partir de ces éléments, l'irradiation et les atteintes qu'elle entraine au niveau du tractus digestif 
pourraient s'ajouter à la liste des diverses situations générant des atteintes multiples. La spécificité des lésions radio-induites vient en partie de la perte cellulaire, irréversible ou non, effective au niveau de différents organes. Cette « signature » des effets de l'irradiation vient renforcer la complexité des processus physiologiques et des réponses organiques qui se déclenchent lors d'une atteinte multi-viscérales. En cas de surexposition accidentelle, qu'elle soit totale ou localisée, les effets radioinduits sur l'hémato-/lymphopoïèse vont vraisemblablement influencer la balance qui existe entre les réponses inflammatoires/anti-inflammatoires de l'intestin, soit de façon bénéfique en limitant le niveau d'infection, soit de façon délétère, en engendrant une réponse inflammatoire incontrôlée. D'un point de vue thérapeutique, il semble ainsi difficile d'envisager la mise en place d'un traitement adapté aux atteintes digestives seules, du fait des communications inter-organes très étroites. Ainsi, l'objectif thérapeutique devra non seulement permettre de protéger et/ou restaurer l'intégrité et la fonctionnalité de la barrière intestinale, mais devra également prendre en compte les atteintes vasculaires et/ou lymphatiques qui y sont associées. Si l'utilisation d'une combinaison de molécules semble évidente, le contrôle des processus inflammatoires et immunitaires nous semble essentiel.

De part sa radiosensibilité importante, et du fait des nombreuses similitudes qui ont pu être trouvées avec les autres cas d'atteintes multi-organes, l'intestin se pose sûrement comme un acteur principal dans le tableau clinique du SDMV radioinduit. Vu le rôle majeur qu'il peut y jouer, le système digestif pourrait être considéré comme une sorte de «sonnette d'alarme » vis-à-vis des méfaits de l'irradiation et une évaluation non invasive, mais représentative, des lésions à cet organe apporterait des éléments d'information essentiels pour améliorer le diagnostic des personnes irradiées.

Remerciements. Ce travail a pu être réalisé en partie grâce à la Délégation générale pour l'armement (DGA, contrat de recherche $n^{\circ}$ 0234033). Cette revue s'appuie sur une conférence présentée lors du congrès de Reisensburg en Allemagne (2003), intitulée "Pathogenetic mechanism in different systems contributing to multi-organ involvment or failure».

\section{RÉFÉRENCES}

Adams C.A., Sambol T., Xu D.Z., Ly Q., Granger D.N., Deitch E.A. (2001) Hemorrhagic shock induced upregulation of P-selectin expression is mediated by factors in mesenteric lymph and blunted by mesenteric lymph duct interruption, J. Trauma 51, 625-631.

Anjaria D.J., Rameshwar P., Deitch E.A., Xu D.Z., Adams C.A., Forsythe R.M., Sambol J.T., Hauser C.J., Livingston D.H. (2001) Hematopoietic failure after hemorrhagic shock is mediated partially through mesenteric lymph, Crit. Care Med. 29, 1780-1785.

Anno G.H., Baum S.J., Withers H.R., Young R.W. (1989) Symptomatology of acute radiation effects in human after exposure to doses of 0.5-30 Gy, Health Phys. 56, 821-838. 
Baranov A.E. (1994) Allogeneic bone marrow transplantation after severe uniform total body irradiation: experience from recent (Nyasvizh, Belarus) and previous radiation accidents, $A d v$. Biosci. 94, 281-293.

Baue A.E. (1996) MOF/MODS, SIRS: an update, Shock 6, S1-S5.

Becciolini A., Balzi M., Potten C.S. (1995) Radiation effects on proliferation and differentiation in the rat small intestine, in Radiation and gut (C.S. Potten, J.H. Hendry, Eds.) pp. 85-143. Elsevier Science, Amsterdam.

Bion J.F. (1999) Is the gut responsible for multiple organ failure? Schweiz Med. Wochenschr. 129, 1600-1604.

Bone R.C. (1996) Immunologic dissonance. A continuing evolution in our understanding of the systemic inflammatory response syndrome (SIRS) and the multiple organ dysfunction syndrome (MODS), Ann. Intern. Med. 125, 686-687.

Buell M.G., Harding R.K. (1989) Proinflammatory effects of local abdominal irradiation on rat gastrointestinal tract, Dig. Dis. Sci. 34, 390-399.

Bush K.T., Keller S.H., Nigam S.K. (2000) Genesis and reversal of ischaemic phenotype in epithelial cells, J. Clin. Invest. 106, 621-626.

Chen L.W., Hsu C.M., Cha M.C., Chen J.S., Chen S.C. (1999) Changes in gut mucosal nitric oxide synthase (NOS) activity after thermal injury and its relation with barrier failure, Shock 11, 104110.

Cho C.H., Kammerer R.A., Lee H.J., Yasunaga K., Kim K.T., Choi H.H., Kim W., Kim S.H., Park S.K., Lee G.M., Koh G.Y. (2004) Designed angiopoietin-1 variant, COMP-Ang1, protects against radiation-induced endothelial cell apoptosis, Proc. Natl. Acad. Sci. USA 101, 55535558.

Cockerham L.G., Doyle T.F., Trumbo R.B., Nold J.B. (1984) Acute post-irradiation canine intestinal blood flow, Int. J. Radiat. Biol. Relat. Stud. Phys. Chem. Med. 45, 65-72.

De Both N.J., Verney M. (1976) Epithelial regeneration of transposed intestine after high doses of Xirradiation, Int. J. Radiat. Res. 29, 17-26.

Debbage P.L., Seidl S., Kreczy A., Hutzler P., Pavelka M., Lukas P. (2000) Vascular permeability and hyperpermeability in a murine adenocarcinoma after fractionated radiotherapy: an ultrastructural tracer study, Histochem. Cell Biol. 114, 259-275.

Deitch E.A. (1992) Multiple organ failure. Pathophysiology and potential future therapy, Ann. Surg. 216, 117-134.

Deitch E.A. (2001) Role of the gut lymphatic system in multiple organ failure, Curr. Opin. Crit. Care 7, 92-98.

Deitch E.A. (2002) Bacterial translocation or lymphatic drainage of toxic products from the gut: What is important in human beings? Surgery 131, 241-244.

Deitch E.A., Xu D., Franko L., Ayala A., Chaudry I.H. (1994) Evidence favoring the role of the gut as a cytokine-generating organ in rats subjected to haemorrhagic shock, Shock 1, 141-145.

Eddy H.A., Casarett G.W. (1968) Intestinal vascular changes in the acute radiation syndrome, in Gastrointestinal Radiation Injury (M.F. Sullivan, Ed.) pp. 385-395. Amsterdam Excerpta Medica Foundation.

Faries P.L., Simon R.J., Martella A.T., Lee M.J., Machiedo G.W. (1998) Intestinal permeability correlates with severity on injury in trauma patients, J. Trauma 44, 1031-35.

Flynn D.F., Mihalakis I., Mauceri T., Pins M.R. (1995) Gastrointestinal syndrome after accidental exposure during radiotherapy, in Radiation and the Gastrointestinal Tract (A. Dubois, G.L. King, D.R. Livengood, Eds.) pp. 225-234. CRC press.

Freeman S.L., Mac Naughton W.K. (2000) Ionizing radiation induces iNOS-mediated epithelial dysfunction in the absence of an inflammatory response, Am. J. Physiol. Gastrointest. Liver Physiol. 278, G243-G250. 
Gianotti L., Alexander J.W., Pyles T., James L., Babcock G.F. (1993) Relationship between extent of burn injury and magnitude of microbial translocation from the intestine, J. Burn Care Rehab. 14, 336-342.

Goris R.J., Beokhorst P.A., Nuytinck K.S. (1985) Multiple organ failure: generalized autodestructive inflammation, Arch. Surg. 120, 1109-1115.

Griffiths N.M., Lebaron-Jacobs L. (2001) Place du syndrome gastro-intestinal dans la radiopathologie accidentelle, in Journée scientifique d'information sur "les lésions radio-induites de l'intestin », EDF-Service de radioprotection, $\mathrm{n}^{\circ} 18$, pp. 4-7.

Guan J., Jin D.D., Jin L.J., Lu Q. (2002) Apoptosis in organs of rats in early stage after polytrauma combined with shock, J. Trauma 52, 104-111.

Guo W., Ding J., Huang Q., Jerrells T., Deitch E.A. (1995) Alterations in intestinal bacterial flora modulate the systemic cytokine response to hemorrhagic shock, Am. J. Physiol. Gastrointest. Liver Physiol. 269, G827-G832.

Haimovitz-Friedman A., Cordon-Cardo C., Bayoumy S., Garzotto M., McLoughlin M., Gallily R., Edwards C.K., Schuchman E.H., Fuks Z., Kolesnick R. (1997) Lipopolysaccharide induces disseminated endothelial apoptosis requiring ceramide generation, J. Exp. Med. 186, 18311841.

Harari Y., Weisbrodt N.W., Moody F.G. (2000) Ileal mucosal response to bacterial toxin challenge, $J$. Trauma 49, 306-313.

Hirama T., Tanosaki S., Kandatsu S., Kuroiwa N., Kamada T., Tsuji H., Yamada S., Katoh H., Yamamoto N., Tsujii H., Suzuki G., Akashi M. (2003) Initial medical management of patients severely irradiated in the Tokai-mura criticality accident, Br. J. Radiol. 76, 246-253.

Husain K.D., Coopersmith C.M. (2003) Role of intestinal apoptosis in survival, Curr. Opin. Crit. Care 9, 159-163.

IAEA (1996) The Radiological Accident at the Irradiation Facility in Nesvizh, Vienna 1996, pp. 4665.

Kaiser V.L., Sifri Z.C., Dikdan G.S., Berezina T., Zaets S., Lu Q., Xu D., Deitch E.A. (2005) Traumahemorrhagic shock mesenteric lymph from rat contains a modified form of albumin that is implicated in endothelial cell toxicity, Shock 23, 417-425.

Kale, I.T., Kuzu M.A., Berkem H., Berkem R., Acar N. (1998) The presence of hemorrhagic shock increases the rate of bacterial translocation in blunt abdominal trauma, J. Trauma Inj. Infect. Crit. Care 44, 171-174.

Koike K., Moore E.E., Moore F.A., Read R.A., Carl V.S., Banerjee A. (1994) Gut ischemia/reperfusion produces lung injury independent of endotoxin, Crit. Care Med. 22, 1438-1444.

Kompan L., Kremzar B., Gadzijev E., Prosek M. (1999) Effects of early enteral nutrition on intestinal permeability and the development of multiple organ failure after multiple injury, Intensive Care Med. 25, 157-161.

Kuebler J.F., Toth B., Rue L.W., Bland K.I., Chaudry I.H. (2003) Differential alterations in intestinal permeability after trauma-hemorrhage, J. Surg. Res. 112, 198-204.

Lamerton L.F., Lord B.I. (1964) Studies of cell proliferation under continuous irradiation, Nat. Cancer Inst. Monogr. 14, 185-198.

Linard C., Ropenga A., Vozenin-Brotons C., Chapel A., Mathe D. (2003) Abdominal irradiation increases inflammatory cytokine expression and activates NF-kappaB in rat ileal muscularis layer, Am. J. Physiol. Gastrointest. Liver Physiol. 285, G556-G565.

Lu Q., Xu D.Z., Davidson M.T., Hasko G., Deitch E.A. (2004) Hemorrhagic shock induces endothelial cell apoptosis, which is mediated by factors contained in mesenteric lymph, Crit. Care Med. 32, 2464-2470.

Magnotti L.J., Xu D.Z., Deitch E.A. (1999) Gut-derived mesenteric lymph. A link between burn and lung injury, Arch. Surg. 134, 1333-1341. 
Maj J.G., Paris F., Haimovitz-Friedman A., Venkatraman E., Kolesnick R., Fuks Z. (2003) Microvascular function regulates intestinal crypt response to radiation, Cancer Res. 63, 43384341.

Mallick I.H., Yang W., Winslet M.C., Seifamian A.M. (2004) Ischemia-reperfusion injury of the intestine and protective strategies against injury, Dig. Dis. Sci. 49, 1359-1377.

Messick W.J., Koruda M., Meyer A., Zimmerman K. (1994) Differential changes in intestinal permeability following burn injury, J. Trauma 36, 306-312.

Monti P., Van der Meeren A., Wysocki J., Griffiths N.M. (2004) Abdominal irradiation: a model of severe trauma and inflammation, Shock 21, 154-613A.

Moore F.A. (1999) Role of gut hypoperfusion in the development of Systemic Inflammatory Response Syndrome (SIRS) and Multiple Organ Failure (MOF), Am. J. Surg. 178, 449-453.

Moore F.A., Moore E.E., Poggetti R., McAnena O., Peterson V., Abernathy C.M., Parsons P.E. (1991) Gut bacterial translocation via the portal vein: A clinical perspective with major torso trauma, J. Trauma 31, 629-638.

Nejdfors P., Ekelund M., Westrom B.R., Willen R., Jeppsson B. (2000) Intestinal permeability is increased after radiation therapy, Dis. Colon Rectum 43, 1582-1588.

Panès J., Anderson D.C., Miyasaka M., Granger D.N. (1995) Role of leukocyte-endothelial cell adhesion in radiation-induced microvascular dysfunction in rats, Gastroenterology 108, 17611769.

Pape H.C., Dwenger A., Regel G., Auf'M'Kolck M., Gollub F, Wisner D., Sturm J.A., Tscherne H. (1994) Increased gut permeability after multiple trauma, Br. J. Surg. 81, 850-852.

Paris F., Fuks Z., Kang A., Capodieci P., Juan G., Ehleiter D., Haimowitz-Friedman A., Cordon-Cardo C., Kolesnick R. (2001) Endothelial apoptosis as the primary lesion initiating intestinal radiation damage in mice, Science 293, 293-297.

Potten C.S. (1990) A comprehensive study of the radiobiological response of the murine (BDF1) intestine, Int. J. Radiat. Biol. 58, 925-973.

Potten C.S. (1995) Effects of radiation on murine gastrointestinal cell proliferation, in Radiation and Gut (C.S. Potten, J.H. Hendry, Eds.) pp. 61-84. Elsevier Science, Amsterdam.

Potten C.S., Grant H.K. (1998) The relationship between ionising radiation-induced apoptosis and stem cells in the small and large intestine, Br. J. Cancer 78, 993-1003

Reidy J.J., Ramsey G. (1990) Clinical trials of selective decontamination of the digestive tract: a review, Crit. Care Med. 18, 1449-1456.

Roumen R.M., Hendricks T., Wevers R.A., Gories J.A. (1993) Intestinal permeability after severe trauma and hemorrhagic shock is incresased without relation to septic complications, Arch. Surg. 125, 453-457.

Ryan C.M., Yarmush M.L., Burke J.F., Tompkins R.G. (1992) Increased gut permeability early after burns correlates with the extent of injury, Crit. Care Med. 20, 1508-1512.

Sambol J.T., White J., Horton J.W., Deitch E.A. (2002) Burn-induced impairment of cardiac contractile function is due to gut-derived factors transported in mesenteric lymph, Shock 18, 272-276.

Solheim K.E., Laerum F., Stordahl A., Aase S. (1991) Urinary excretion of iohexol after enteral administration in rats with radiation injury of the small intestine, Scand. J. Gastroenterol. 26, 1097-1106.

Spitzer T.R. (1995) Clinical aspects of irradiation-induced alimentary tract injury, in Radiation and the Gastrointestinal tract (A. Dubois, G.L. King, D.R. Livengood, Eds.) pp. 3-20. CRC Press, Boca Raton.

Tani T., Fujino M., Hanasawa K., Shimizu T., Endo Y., Kodama M. (2000) Bacterial translocation and tumor necrosis factor-alpha gene expression in experimental hemorrhagic shock, Crit. Care Med. 28, 3705-3709. 
Tawadrous Z.S., Delude R.L., Fink M.P. (2002) Resuscitation from hemorrhagic shock with Ringer's ethyl pyruvate solution improves survival and ameliorates intestinal mucosal hyperpermeability in rats, Shock 17, 473-477.

Thiagarajah J., Gourmelon P., Griffiths N.M., Lebrun F., Naftalin R.J., Pedley K.C. (2000) Radiationinduced cytochrome $\mathrm{c}$ release causes loss of colonic fluid absorption by damage to crypts and pericryptal myofibroblasts, Gut 47, 675-684.

Van der Meeren A., Mouthon M.A., Vandamme M., Squiban C., Aigueperse J. (2004) Combinations of cytokines promote mouse survival and limit acute radiation damage in concert with amelioration of vascular damage, Radiat. Res. 161, 549-559.

Van der Meeren A., Monti P., Vandamme M., Squiban C., Wysocki J., Griffiths N. (2005) Abdominal radiation exposure elicits inflammatory responses and abscopal effects in the lungs of mice, Radiat. Res. 163, 144-152.

Varedi M., Greeley G.H., Herndon D.N., Englander E.W. (1999) A thermal injury-induced circulating factor(s) compromises intestinal cell morphology, proliferation and migration, Am. J. Physiol. Gastrointest. Liver Physiol. 277, G175-G182.

Varedi M., Chinery R., Greeley G.H., Herndon D.N., Englander E.W. (2001) Thermal injury effects on intestinal crypt cell proliferation and death are cell position dependent, Am. J. Physiol. Gastrointest. Liver Physiol. 280, G157-G163.

Vigneulle R.M. (1995) Nearby shielding influences survival of the irradiated intestine, in Radiation and the Gastrointestinal tract (A. Dubois, G.L. King, D.R. Livengood, Eds.) pp. 161-170. CRC Press, Boca Raton.

Vigneulle R.M., Rao S., Fasano A., MacVittie T.J. (2002) Structural and functional alterations of gastrointestinal tract following radiation-induced injury in the rhesus monkey, Dig. Dis. Sci. 47, 1480-1491.

Wang W., Smail N., Wang P., Chaudry I.H. (1998) Increased gut permeability after hemorrage is associated with upregulation of local and systemic IL-6, J. Surg. Res. 79, 39-42.

Willoughby D.A. (1960) Pharmacological aspects of the vascular permeability changes in the rat's intestine following abdominal irradiation, Br. J. Radiol. 23, 515-519.

Zallen G., Moore E.E., Johnson J.L., Tamura D.Y., Ciesla D.J., Silliman C.C. (1999) Post hemorrhagic shock mesenteric lymph primes circulating neutrophils and provokes lung injury, J. Surg. Res. 83, 83-88.

Ziegler T.R., Smith R.J., O’Dwyer S.T., Demling R.H., Wilmore D.W. (1988) Increased intestinal permeability associated with infection in burn patients, Arch. Surg. 123, 1313-1319. 